Annales Geophysicae (2002) 20: 1743-1750 (C) European Geosciences Union 2002

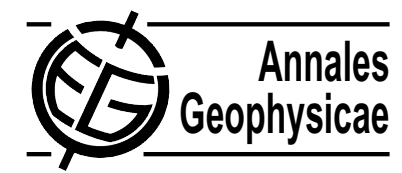

\title{
Altitude dependence of plasma density in the auroral zone
}

\author{
P. Janhunen ${ }^{1}$, A. Olsson ${ }^{2}$, and H. Laakso ${ }^{3}$ \\ ${ }^{1}$ Finnish Meteorological Institute, Geophysical Research, Helsinki, Finland \\ ${ }^{2}$ Swedish Institute of Space Physics, Uppsala Division, Uppsala, Sweden \\ ${ }^{3}$ ESTEC, Space Science Department, Noordwijk, The Netherlands
}

Received: 21 February 2002 - Revised: 5 June 2002 - Accepted: 2 July 2002

\begin{abstract}
We study the altitude dependence of plasma depletions above the auroral region in the $5000-30000 \mathrm{~km}$ altitude range using five years of Polar spacecraft potential data. We find that besides a general decrease of plasma density with altitude, there frequently exist additional density depletions at $2-4 R_{E}$ radial distance, where $R_{E}$ is the Earth radius. The position of the depletions tends to move to higher altitude when the ionospheric footpoint is sunlit as compared to darkness. Apart from these cavities at $2-4 R_{E}$ radial distance, separate cavities above $4 R_{E}$ occur in the midnight sector for all $K_{p}$ and also in the morning sector for high $K_{p}$. In the evening sector our data remain inconclusive in this respect. This holds for the ILAT range 68-74. These additional depletions may be substorm-related. Our study shows that auroral phenomena modify the plasma density in the auroral region in such a way that a nontrivial and interesting altitude variation results, which reflects the nature of the auroral acceleration processes.
\end{abstract}

Key words. Magnetospheric physics (auroral phenomena; magnetosphere-ionosphere interactions)

\section{Introduction}

Plasma density appears to be considerably variable in the auroral zone. While crossing that region, satellites commonly observe deep density depletions of various spatial and temporal scales. In this paper we use the word cavity as meaning the same as a density depletion, i.e., an occurrence of low plasma density in an absolute sense, without regard to how spatially or temporally localized it is. In addition to ionospheric plasma density depletions (Doe et al., 1993), density depletions often occur in the nightside auroral region at a few thousand kilometer altitudes (Benson and Calvert, 1979; Calvert, 1981) and above (Persoon et al., 1988). There is a correlation between the depletions and upflowing ions and precipitating auroral electrons (Persoon et al., 1988; Per-

Correspondence to: P. Janhunen (Pekka.janhunen@fmi.fi) soon, 1988), thus the density depletions are probably intimately related to the inverted- $\mathrm{V}$ and auroral arc formation as has also been demonstrated using simulations (e.g. Ergun et al., 2000). The density depletions are also suggested to be the source regions of the auroral kilometric radiation (AKR) (Strangeway et al., 2001). The association with inverted-V phenomena whose scale sizes are usually less than $100 \mathrm{~km}$ is also in line with the facts that the deepest depletions typically have scale sizes less than $100 \mathrm{~km}$ (Hilgers, 1992) and that these depletions are most common in the nightside. Density depletions of more than $40 \%$ of the background density are observed in 70\% of the nightside crossings of DE-1 (Persoon et al., 1988). Density depletions with AKR are often stable for at least several tens of minutes (Bahnsen et al., 1989).

The purpose of this paper is to study statistically the altitude variation $\left(1.5-6 R_{E}\right.$ radial distance, where by radial distance we mean the direct distance from the center of the Earth) of plasma density in the auroral zone, using five years (1996-2001) of spacecraft potential data from the Electric Field Investigation (EFI) onboard Polar (Harvey et al., 1995). We will study this density variation as a function of magnetic local time (MLT) sectors, invariant latitude (ILAT), $K_{p}$ index and solar illumination. A statistical study on auroral density depletions at $1 R_{E}$ altitude has been published recently, using one year of measurements by the same instrument (Johnson et al., 2001).

\section{Observations}

The basic principle of using the spacecraft potential for studying plasma density variations is as follows (Pedersen, 1995; Laakso and Pedersen, 1998; Johnson et al., 2001): when a satellite, immersed in a tenuous plasma (density below $1000 \mathrm{~cm}^{-3}$ ), is illuminated by the Sun, continuous photoelectron emission from the surface of the spacecraft causes the satellite to become positively charged with respect to the surrounding plasma. An equilibrium is reached when the thermal flux of electrons from the surrounding plasma equals 
the photoelectron flux. It can be shown that the spacecraft potential is larger (more positive) when the plasma density is small. The exact relationship between the spacecraft potential and the density remains uncertain because it depends on the details of the photoelectron energy spectrum and thus on the properties of the satellite surface material and solar spectrum, although one can calibrate the relationship by using comparisons with more direct ways to calculate the plasma density (Scudder et al., 2000). In the present study we assume that a spacecraft potential versus density relationship exists, but knowing the relationship in detail is not necessary to draw our conclusions.

In this study we use the Polar/EFI spacecraft potential measurements, sampled at 2.5 times per second. To keep the statistical analysis to a manageable size we take every 30 th sample of the raw data, thus yielding one data point every $12 \mathrm{~s}$. We follow the usual convention and always display the negative of the spacecraft potential. Eclipse periods are removed because in those the spacecraft potential is not an indicator of plasma density. We call the negative of the spacecraft potential $V_{\mathrm{SC}}$, measured in volts. To get information about density variations we selected three threshold values for $V_{\mathrm{SC}}$ : $-11 \mathrm{~V},-18 \mathrm{~V}$ and $-25 \mathrm{~V}$ (corresponding to densities of roughly $0.3 \mathrm{~cm}^{-3}, 0.1 \mathrm{~cm}^{-3}$ and $0.06 \mathrm{~cm}^{-3}$, respectively (Scudder et al., 2000). However, our results are not sensitive to the calibration of the spacecraft potential versus density relationship because only relative changes are considered. We only ask questions like what is the occurrence frequency of $V_{\mathrm{SC}}<-11 \mathrm{~V}$ as a function of altitude etc., without having to know exactly what density value $-11 \mathrm{~V}$ actually corresponds to.

In principle the spacecraft potential depends not only on density but also on electron temperature (Laakso and Pedersen, 1998; Johnson et al., 2001). To evaluate this effect quantitatively we use Eqs. (1)-(4) of Johnson et al. (2001) generalizing them to the case of two Maxwellian electron populations, one representing the background plasma (the cold component) and the other, if any, representing the energetic plasma (the hot component). The Debye length needed in the shielding distance calculation in Eq. (2) of Johnson et al. (2001) is given by $\lambda_{D e}=\left(\lambda_{D e 1}^{-2}+\lambda_{D e 2}^{-2}\right)^{-1 / 2}$ where $\lambda_{D e 1}$ and $\lambda_{D e 2}$ are the cold and hot component Debye lengths, respectively.

In Fig. 1 we show the density corresponding to a spacecraft potential value of $-25 \mathrm{~V}$, for varying mixtures of hot and cold plasma. The cold electron temperature is fixed and set to $5 \mathrm{eV}$, and the hot electron temperature is varied between 5 $\mathrm{eV}$ and $10 \mathrm{keV}$. The mixing ratio (hot density versus total density) is varied between $1 \%$ and $90 \%$. When the electron temperatures are below some hundred electronvolts, the total density corresponding to $-25 \mathrm{~V}$ is almost independent of the temperature and density mixing ratio and equal to about 0.05 $\mathrm{cm}^{-3}$ (in the specific model of spacecraft and photoelectrons used by, Johnson et al., 2001). To reach 50\% deviation from this total density value, i.e. density $0.025 \mathrm{~cm}^{-3}$, one should, for example, have $35 \%$ of $2 \mathrm{keV}$ plasma.

Figure 2 is rather similar to Fig. 1, but now we keep the

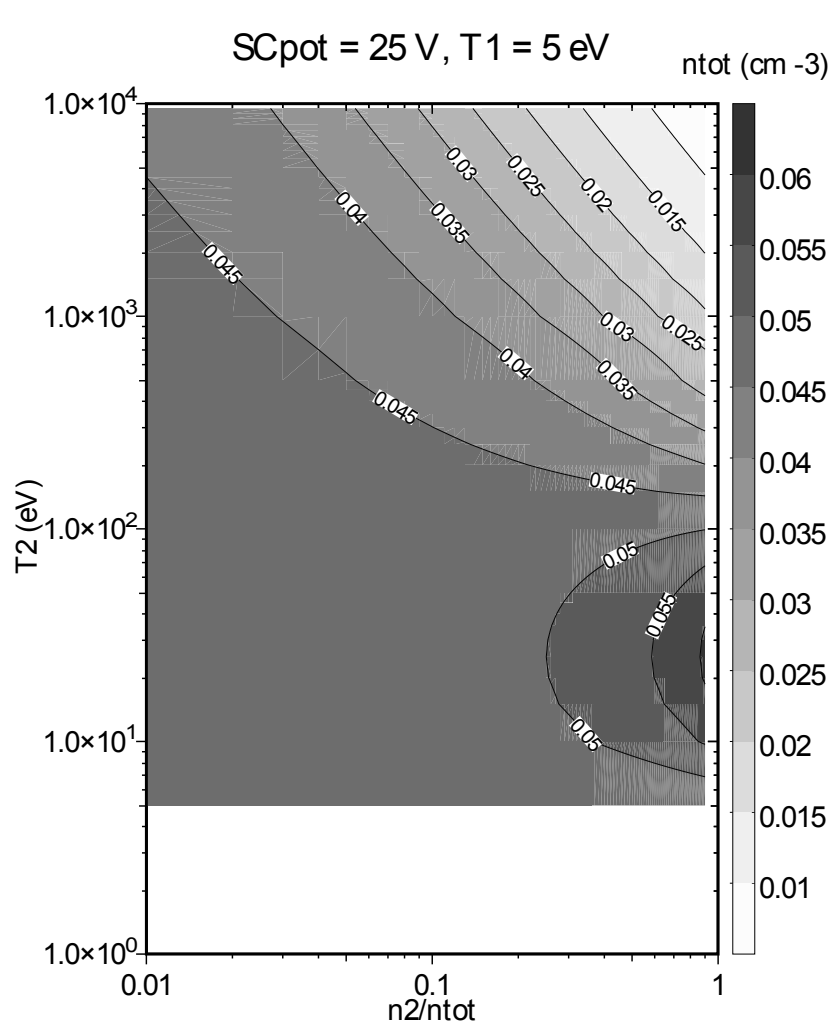

Fig. 1. Density value corresponding to a spacecraft potential $-25 \mathrm{~V}$ in a model containing two Maxwellian electron populations, called hot and cold. The horizontal axis is the hot versus total density ratio and the vertical axis is the hot component temperature. The cold component temperature is set to $5 \mathrm{eV}$.

total density fixed at $0.05 \mathrm{~cm}^{-3}$ and plot the spacecraft potential for different hot temperatures and mixing ratios. From Figs. 1 and 2, we can draw the conclusion that for the given densities, the increase of the temperature of the hot component increases Vsc, as expected, but the change is not very dramatic unless we have only a hot population and its temperature approaches $1 \mathrm{keV}$ and goes beyond that. More specifically, a region with $0.05 \mathrm{~cm}^{-3}$ density (a "deep" cavity), which normally should have the spacecraft potential about $-25 \mathrm{~V}$, may have a spacecraft potential of only $-18 \mathrm{~V}$ and thus be classified only "medium-deep" if it contains $20 \%$ of $10 \mathrm{keV}$ electrons. In practice, the temperature should not have a significant effect on our statistical results, except perhaps within and below the bottom of the acceleration region (below 2-3 $R_{E}$ radial distance, depending on the solar illumination condition of the footpoint ionosphere) where some cavities might be missed because of the abundance of highenergy inverted-V electrons.

We stress that although we for definiteness use a specific model (Johnson et al., 2001) to compute explicit density values, we do not need the exact spacecraft potential versus density relationship to reach the conclusions of this study, but only the relative changes of this relationship are of importance. 


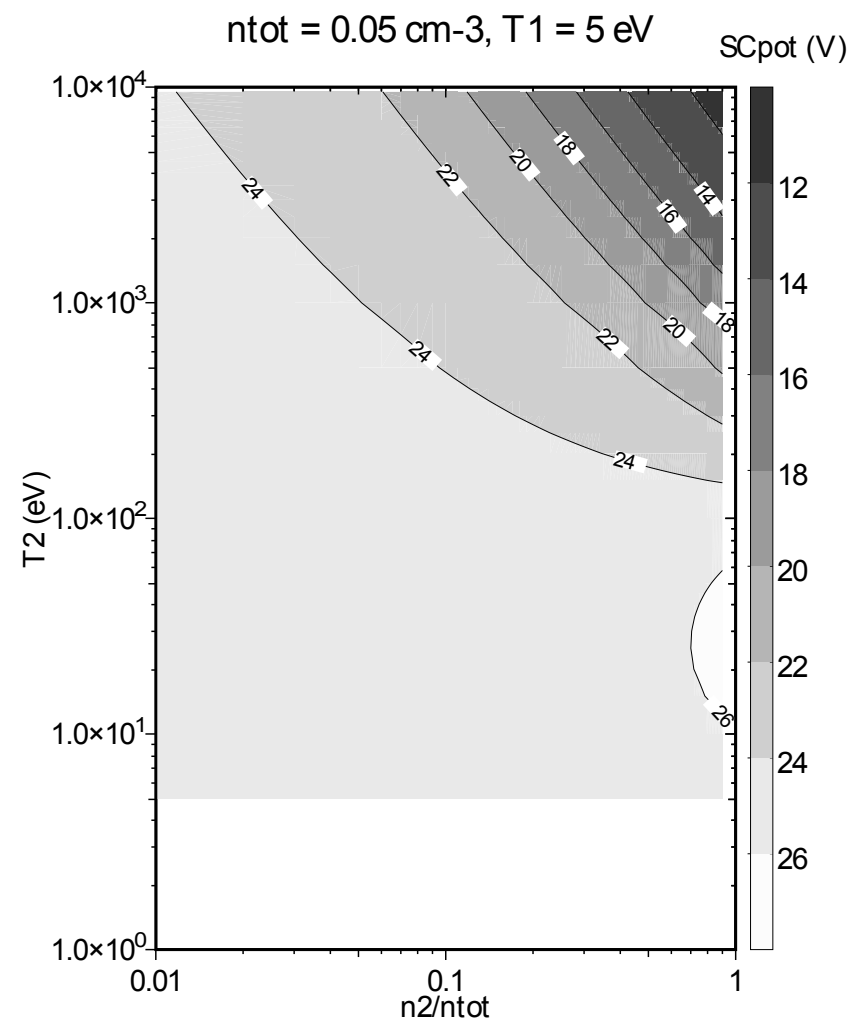

Fig. 2. Spacecraft potential value corresponding to density 0.05 $\mathrm{cm}^{-3}$ in a model containing two Maxwellian electron populations, called hot and cold. The horizontal axis is the hot versus total density ratio and the vertical axis is the hot component temperature. The cold component temperature is set to $5 \mathrm{eV}$.

\section{Results}

For an overview, we show in Fig. 3 the dependence of cavity occurrence rate on MLT and ILAT for threshold values $-11 \mathrm{~V},-18 \mathrm{~V}$ and $-25 \mathrm{~V}$. The occurrence rate is defined as the number of data points where the spacecraft potential is more negative than the given threshold, divided by the total number of data points in the $R$, MLT, ILAT, etc. bin in question. All radial distance bins, including measurements during all magnetic conditions, are put together in this plot. The orbital coverage is also shown in the bottom panel. The occurrence rate of large satellite potentials, or low plasma densities, follows the general pattern of the auroral oval (Feldstein and Starkov, 1967) in the nightside MLT sectors. (In the dayside MLT sectors the mean oval is poleward of 74 ILAT and so cannot be seen in our plot). This result is in agreement with Johnson et al. (2001). The lowest densities (highest occurrence rate for cavities) are found above ILAT 68.

In Fig. 4 we show the occurrence frequencies of auroral cavities at 18:00-06:00 MLT sector for the cases where the ionospheric footpoint is in darkness (left subplot) or sunilluminated (right subplot). Again, three different spacecraft potential thresholds are used $(-11 \mathrm{~V},-18 \mathrm{~V}$ and $-25 \mathrm{~V})$ representing shallow, medium-deep and deep cavities, respectively. Geomagnetic activity is binned into two groups so

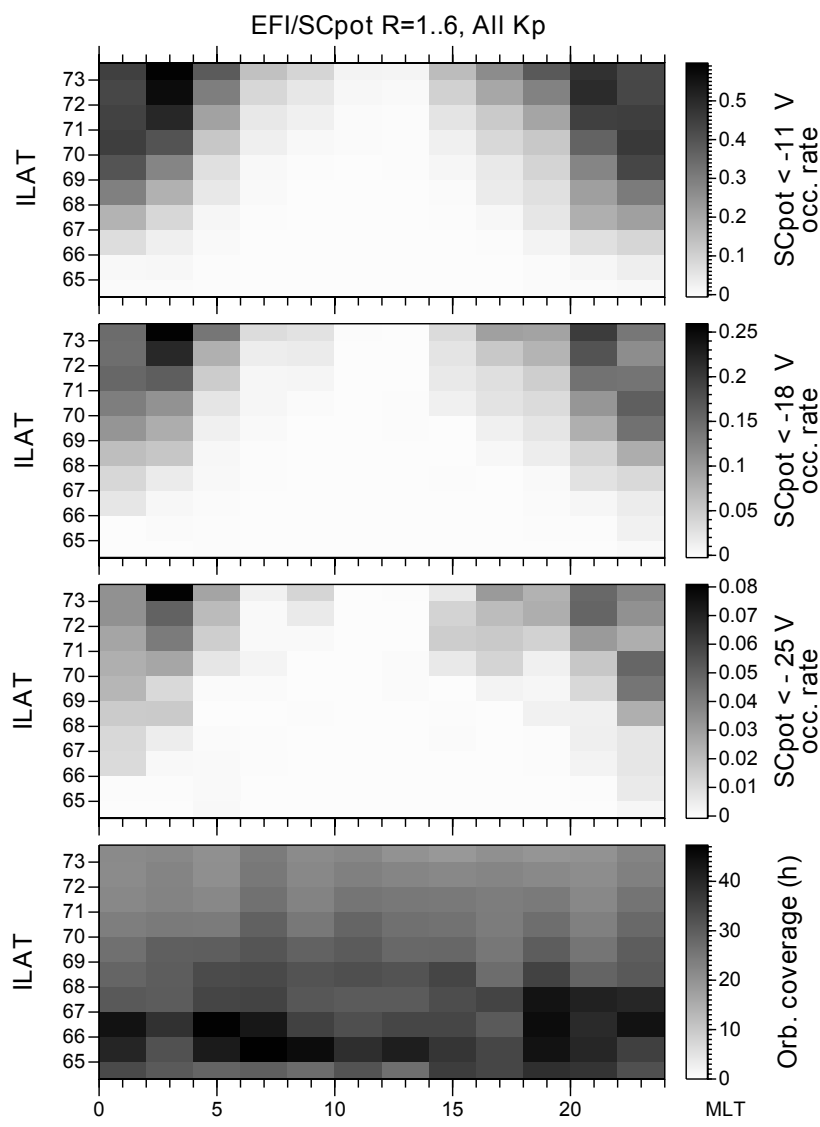

Fig. 3. Occurrence frequency of cavities for three different thresholds as a function of MLT and ILAT. The lowest panel shows the orbital coverage.

that small $K_{p}$ index values $\left(0 \leq K_{p} \leq 2\right)$ are shown by solid lines with filled circles, large $K_{p}$ index values $\left(K_{p}>2\right)$ by solid lines with open triangles, and all $K_{p}$ 's put together by dotted lines with no symbol. The orbital coverage is also shown on the top. The occurrence frequency was first calculated separately for each ILAT bin (the ILAT bin width is $1^{\circ}$ ), then linearly averaged over ILAT. Because of this, the results are not sensitive to possibly nonuniform ILAT coverage. Error bars are shown except when the error is insignificantly small. The error estimates are calculated from the number of orbital crossings visiting the ( $R$, ILAT, MLT) bin. If the number of data points is $N$, the number of points satisfying the cavity threshold condition is $n$ and the number of orbital crossings contributing to the bin is $K$, the occurrence frequency is $f=n / N$ and its standard deviation $\Delta f=\sqrt{f(1-f) / K}$. This method of error estimation considers measurements made during one orbital crossing within the bin as fully correlated. Thus it is likely that the true errors are somewhat smaller than our error bars.

From the second panels of Fig. 4 (shallow cavities, spacecraft potential more negative than $-11 \mathrm{~V}$ ) we see that cavities are rare, i.e., plasma densities are on the average high, close to the Earth and that the region of higher plasma densities extends to a higher altitude when the ionospheric foot- 

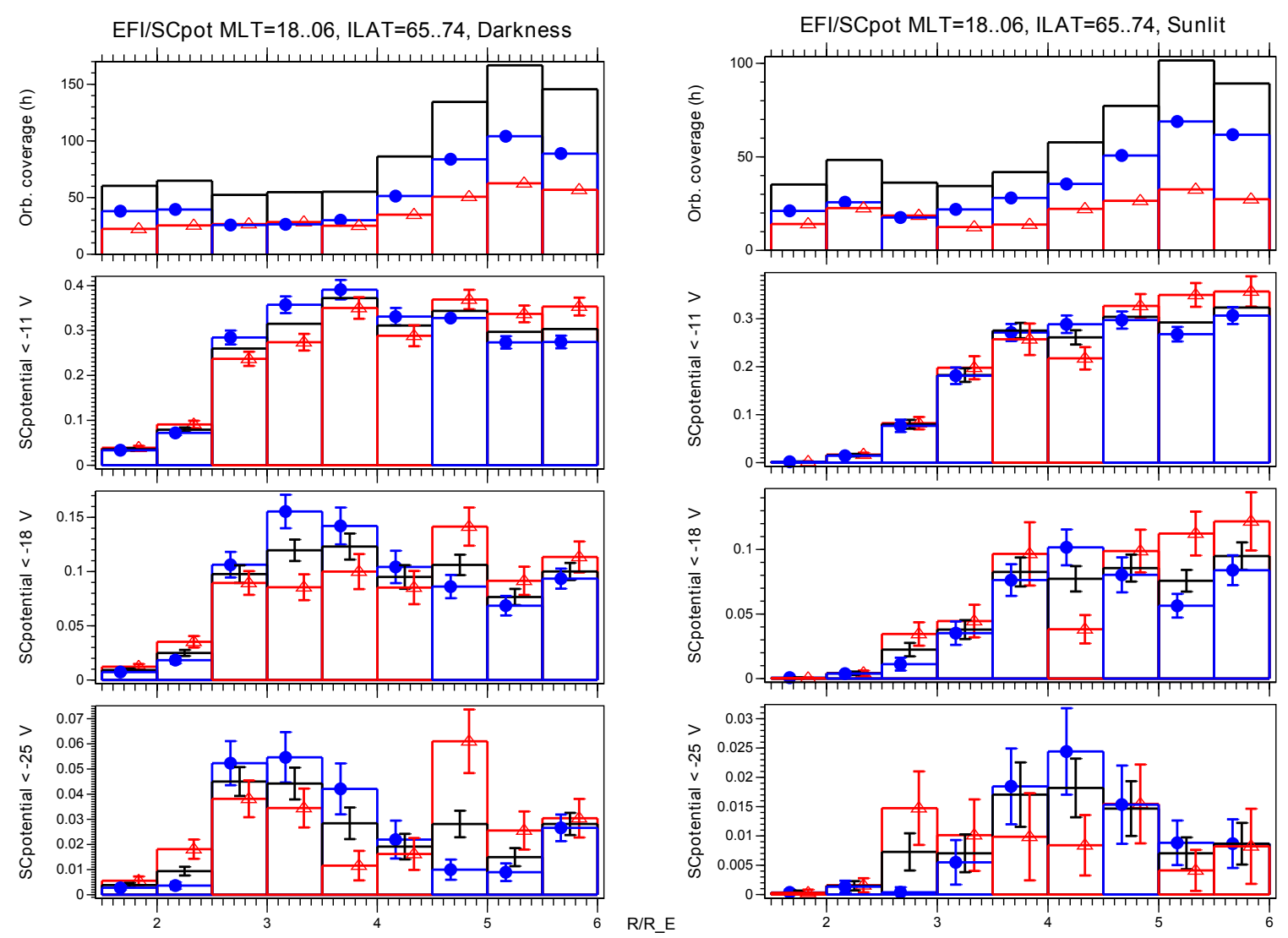

R/R_E

Fig. 4. Occurrence frequency of nightside auroral cavities for conditions when the ionospheric footpoint is in darkness (left subplot) and sun-illuminated (right subplot). The top panel of each subplot is the orbital coverage in hours while the remaining panels give the occurrence frequency for spacecraft potential thresholds $-11 \mathrm{~V},-18 \mathrm{~V}$ and $-25 \mathrm{~V}$. Low $K_{p}(\leq 2)$ is shown by blue lines with filled dots, high $K_{p}(>2)$ by red lines and open triangles and all $K_{p}$ 's put together by solid black line with no symbol. The horizontal axis in each subplot is the radial distance from 1.5 to $6 R_{E}$. Error bars are shown except when they are insignificantly small. If the orbital coverage is less than 20 min, the corresponding occurrence frequencies are not shown.

point is illuminated by the sun. The occurrence frequency of the shallow cavities is almost constant at higher altitudes. Deeper cavities (third and fourth panels) are, on the contrary, concentrated near a specific radial distance. The maximum occurrence frequency is at around 3.25 $R_{E}$ radial distance for darkness conditions and at $4.25 R_{E}$ for sunlit conditions. The solar illumination condition is calculated at the $110 \mathrm{~km}$ altitude. It thus seems that cavities of about $1-1.5 R_{E}$ radial extent tend to exist at this altitude and that their preferred altitude is lifted up by about $1 R_{E}$ when the footpoint is illuminated by the sun.

\subsection{Darkness conditions}

Since the effect of solar illumination is seen to be important, we from now on separate all our statistics in darkness and sunlit conditions. In Fig. 5 we show an MLT and ILAT-decomposed view of the statistics. We have three 4-h MLT sectors (18:00-22:00, 22:00-02:00 and 02:00-06:00) and three 3-degree ILAT ranges $\left(65-68^{\circ}, 68-71^{\circ}\right.$ and $71-$ $74^{\circ}$ ). Notice that the scales are different for all panels due to a large range of values.

The peak in occurrence frequency at around $3 R_{E}$ is seen in many of the subplots of Fig. 5, especially in the premidnight sectors below $71^{\circ}$, and it becomes clearer when the spacecraft potential threshold is made more negative $\left(V_{\mathrm{SC}}<-18 \mathrm{~V}\right.$ and $\left.V_{\mathrm{SC}}<-25 \mathrm{~V}\right)$. The occurrence rate of cavities naturally becomes lower when the threshold is made more negative. Otherwise the altitude of the peak does not seem to depend on the threshold. Also, the peak altitude does not much depend on the $K_{p}$ index.

Comparison of different ILAT ranges (different rows in Fig. 5) shows that cavities are rare for low $K_{p}$ for low ILAT $(65 \ldots 68)$, especially in the evening and morning MLT sectors, while high $K_{p}$ cavities are more common even for low ILAT. This has been inferred earlier in the $2-4 R_{E}$ radial distance range from DE-1 (Persoon, 1988). A natural explanation for this is the expansion of the auroral oval to lower ILAT during disturbed conditions (Feldstein and Starkov, 1967).

The peak altitude is lower by $0.5 R_{E}$ (residing at $2.75 R_{E}$ radial distance) in the midnight sector as compared to the morning sector $\left(3.25 R_{E}\right)$ if one looks at all $K_{p}$ values and 

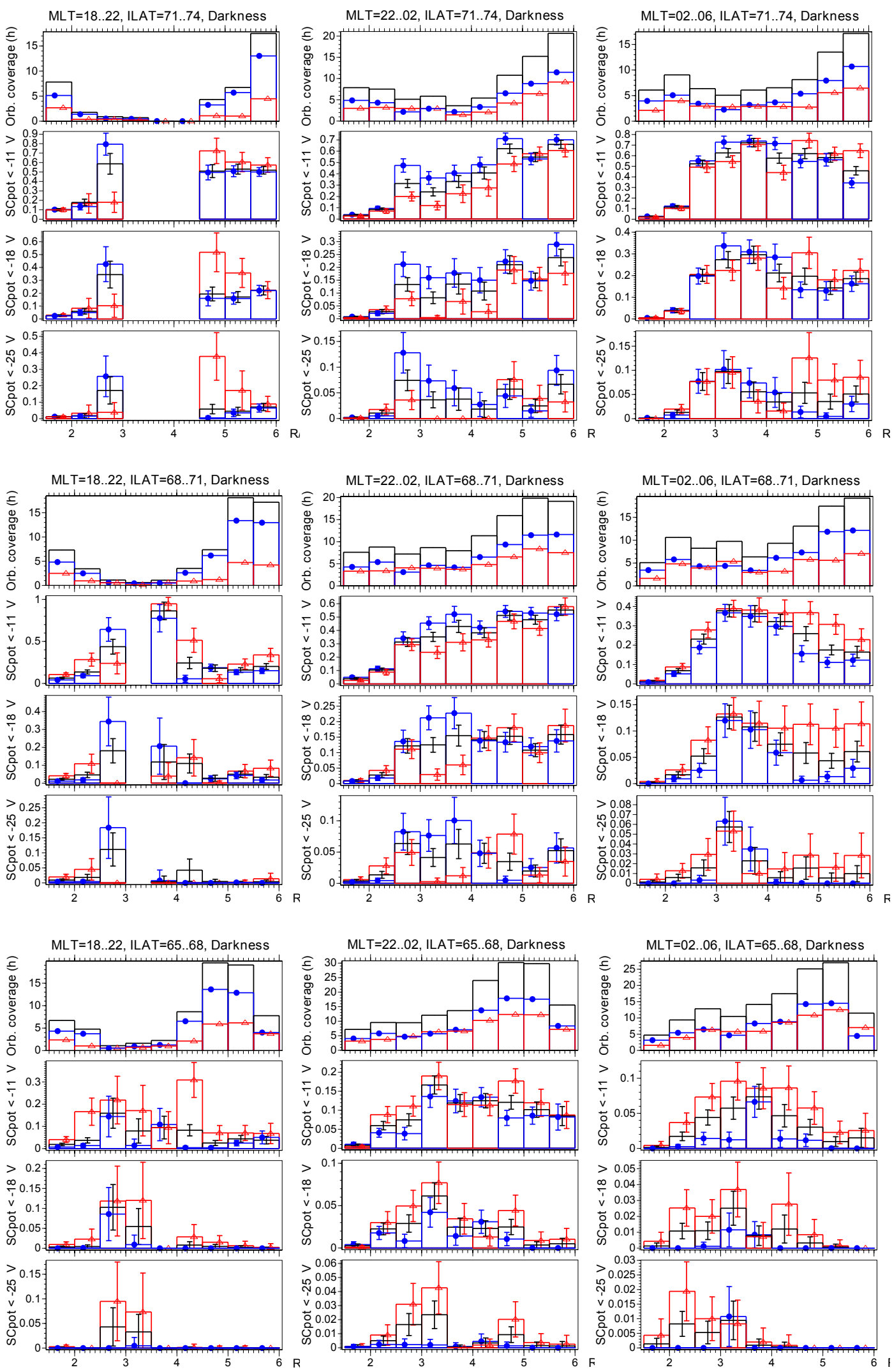

Fig. 5. Occurrence frequency of spacecraft potential being more negative than a threshold. Same format as Fig. 4 but for darkness conditions only and decomposed into three MLT and three ILAT ranges. The MLT and ILAT ranges are shown in the title of each of the nine subplots. MLT changes along the rows and ILAT along the columns. As before, low $K_{p}$ is shown by blue, high $K_{p}$ as red and all $K_{p}$ 's put together by black. 

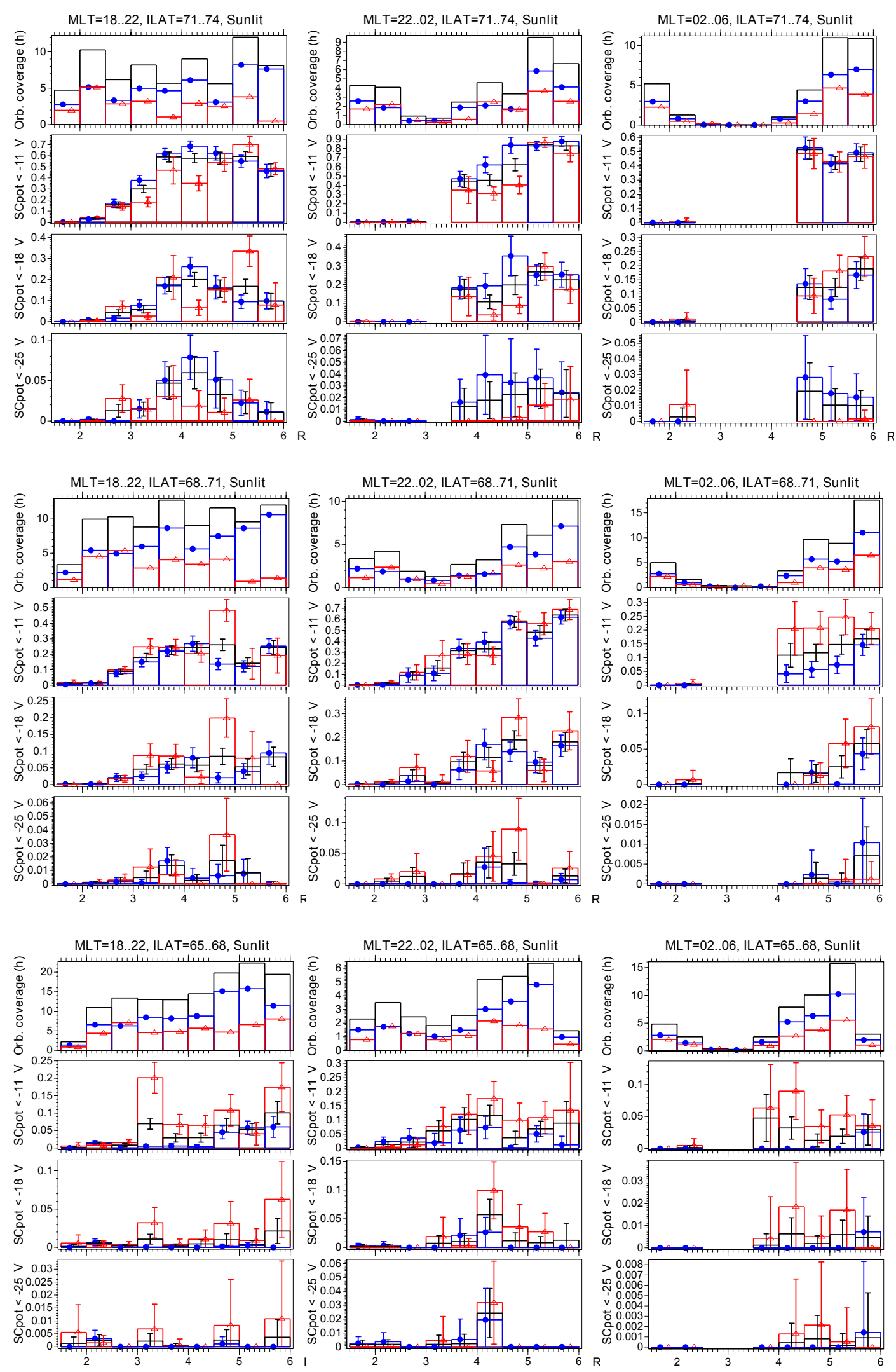

Fig. 6. Same as Fig. 5, but for conditions when the ionospheric footpoint is sun-illuminated. 
at the medium-deep and deep cavities in which the peak is most clearly seen. For the evening sector we cannot make this comparison because of lack of orbital coverage. The tendency of deep cavities to occur only around $3 R_{E}$ radial distance if very strong for low ILAT (65 . . 68).

Apart from the cavities occurring near $3 R_{E}$ radial distance, there are more irregularly occurring cavities also at higher altitude, especially in the midnight sector, but also in the morning sector when the $K_{p}$ index is above 2 and when ILAT $\geq 68$. These high altitude cavities have a tendency of being separated from the lower altitude ones, e.g. in the midnight sector $(22 \ldots 02)$ and in the main auroral oval ILAT $(68 \ldots 71)$ there are no deep cavities at all in the $3.75 R_{E}$ radial bin. Because of their MLT and $K_{p}$ dependence, it seems possible that these high-altitude cavities are substormrelated. To confirm this we would need better coverage in the evening sector, however.

\subsection{Sunlit conditions}

Figures 6 is similar to Fig. 5, but it describes sunlit conditions. The overall occurrence frequency of cavities is not much lower in sunlit conditions than in darkness, except for the deepest $(-25 \mathrm{~V})$ cavities. For sunlit conditions we have good coverage in the evening sector, but not so good in the midnight and morning sectors. In the evening sector there is a clearly defined island of cavities which is best visible in the $-25 \mathrm{~V}$ threshold and $71 \ldots 74$ ILAT bin. In this ILAT bin the island is centered at $4.25 R_{E}$ radial distance. For lower ILAT the island altitude gets a bit lower and it becomes less regular. In the $65 \ldots 68$ ILAT bin there is no longer a clearly defined island, at least not for small $K_{p}$. We think that this island of cavities is the same as the $\sim 3 R_{E}$ occurrence frequency peak seen in the darkness statistics, but which is now raised to higher altitude because of increased ionospheric plasma density. Because of incomplete orbital coverage we do not attempt to analyse to what extent the island exists in sunlit conditions in the midnight and morning MLT sectors.

\section{Summary and discussion}

Our statistical study of density depletions using Polar spacecraft potential reveals the following main conclusions:

1. Cavities show an auroral zone dependency, which indicates that most of them are associated with auroral processes. The low-altitude ( $R \approx 2 R_{E}$ ) part of this result has been found earlier (Johnson et al., 2001).

2. Cavities are raised to $\sim 1 R_{E}$ higher altitude in sunlit conditions as compared to darkness conditions.

3 . In darkness, there is a peak in cavity occurrence frequency at about $3.25 R_{E}$ radial distance. The region of cavities ends at about $4.25 R_{E}$, apart from the midnight sector (see item 7 below). In sunlit conditions the maximum is at $3.75 R_{E}$ and the region of cavities extends up to $5.25 R_{E}$.
4. The peak is clearest for the deepest cavities.

5. The $K_{p}$ index does not have a clear effect on the peak altitude.

6. For low ILAT, cavities occur mainly only for large $K_{p}$, probably because the auroral oval extends equatorward during disturbed conditions. For other ILAT, however, cavities are more common for low $K_{p}$ than for high $K_{p}$. This has been inferred earlier in the $2-4 R_{E}$ radial distance range from DE-1 (Persoon, 1988). A possible reason is that it takes time for an auroral arc-associated cavity to develop low densities, and under disturbed conditions, the arcs may move faster to a new location before a really deep cavity has been set up in the old location.

7. In the midnight sector there are cavities also in the 4$6 R_{E}$ radial distance range for both low and high $K_{p}$. They also occur in the morning sector, but only for high $K_{p}$. This holds for ILAT range 68-74. These cavities may be related to substorms.

8. The influence of the electron energy on the spacecraft potential versus density relationship is such that in our statistics, some cavities might be missed within and below the bottom of the acceleration region (about 2-3 $R_{E}$ radial distance, depending on whether the footpoint is sun-illuminated or not).

The overall behavior of the plasma density in the auroral zone in the 5000-30000 km altitude range thus seems to be that (1) in general, density decreases with altitude, and (2) superposed on this basic trend, auroral density depletions occur, These density depletions have an upper boundary at $\sim 20000 \mathrm{~km}$ altitude, in addition to having a lower boundary below $\sim 10000 \mathrm{~km}$ altitude. As shown above, the average altitudes of the boundaries of the auroral density depletions depend on the solar illumination condition. This is natural, since the increased ionospheric plasma density during sunlit conditions makes it harder to create deep cavities at low altitude.

Our results suggest that density cavities associated with auroral processes do not usually continue beyond $4 R_{E}$ radial distance, except perhaps in substorm-related cases. Since auroral density cavities at low altitude are associated with auroral potential structures (Strangeway et al., 1998), they could also plausibly have a similar relationship with each other at higher altitude. In this light, the recent suggestion that auroral potential structures are primarily confined below $4 R_{E}$ radial distance (Janhunen et al., 1999) is consistent with the density cavity statistics presented here. In a recent work modelling the potential structure above an auroral arc (Janhunen and Olsson, 2000), an O-shaped potential together with a self-consistent maintenance mechanism was suggested. In this model, magnetospheric few hundred electronvolt electrons would be repelled by a downward parallel electric field associated with the upper part of the potential structure. An electron cavity of similar shape as the potential 
would thus be the result of this filtering effect. Ions would move faster within the negative O-potential due to electrostatic forces and thus their density would also be reduced, thus quasineutrality would remain. In other words, the model suggested by (Janhunen and Olsson, 2000) predicts an arcassociated density cavity to exist at a limited altitude range, which is in harmony with the statistical findings reported in the present paper.

Acknowledgements. We are grateful to Forrest S. Mozer for providing the EFI data. The work of PJ was supported by the Academy of Finland and that of AO by the Swedish Research Council.

Topical Editor G. Chanteur thanks two referees for their help in evaluating this paper.

\section{References}

Bahnsen, A., Pedersen, B. M., Jespersen, M., Ungstrup, E., Eliasson, L., Murphree, J. S., Elpinstone, R. D., Blomberg, L., Holmgren, G., and Zanetti, L. J.: Viking observations at the source region of auroral kilometric radiation, J. Geophys. Res., 94, 66436654, 1989.

Benson, R. F. and Calvert, W.: ISIS 1 observations at the source of auroral kilometric radiation, J. Geophys. Res., 94, 6643-6654, 1989.

Calvert, W.: The auroral plasma cavity, Geophys. Res. Lett., 8, 919921, 1981.

Doe, R. A., Mendillo, M., Vickrey, J. F., Zanetti, L. J., and Eastes, R. W.: Observations of nightside auroral cavities, J. Geophys. Res., 98, 293-310, 1993.

Ergun, R. E., Carlson, C. W., McFadden, J. P., Mozer, F. S., and Strangeway, R. J.: Parallel electric fields in discrete arcs, Geophys. Res. Lett., 27, 4053-4056, 2000.

Feldstein, Y. I. and Starkov, G. V.: Dynamics of auroral belt and polar geomagnetic disturbances, Planet. Space Sci., 15, 209-229, 1967.

Harvey, P., et al.: The electric field instrument on the Polar satellite, Space Sci. Rev., 71, 583-596, 1995.

Hilgers, A.: The auroral radiating plasma cavities, Geophys. Res.
Lett., 19, 237-240, 1992.

Janhunen, P., Olsson, A., Mozer, F. S., and Laakso, H.: How does the U-shaped potential close above the acceleration region? A study using Polar data, Ann. Geophysicae, 17, 1276-1283, 1999.

Janhunen, P. and Olsson, A.: New model for auroral acceleration: O-shaped potential structure cooperating with waves, Ann. Geophysicae, 18, 596-607, 2000.

Johnson, M. T., Wygant, J. R., Cattell, C., Mozer, F. S., Temerin, M., and Scudder, J.: Observations of the seasonal dependence of the thermal plasma density in the southern hemisphere auroral zone and polar cap at $1 R_{E}$, J. Geophys. Res., 106, $19023-$ $19033,2001$.

Laakso, H. and Pedersen, A.: Ambient electron density derived from differential potential measurements, in: Measurement Techniques in Space Plasmas, (Eds) Borovsky, J., Pfaff, R., and Young, D., AGU Monograph 102, pp. 49-54, AGU, Washington, DC, 1998.

Lin, C. S. and Hoffman, R. A.: Observations of inverted-V electron precipitation, Space Sci. Rev., 30, 415-457, 1982.

Mäkela, J. S., Mälkki, A., Koskinen, H. E. J., Boehm, M., Holback, B., and Eliasson, L.: Observations of mesoscale auroral plasma cavity crossings with the Freja satellite, J. Geophys. Res., 103, 9391-9404, 1998.

Pedersen, A.: Solar wind and magnetospheric plasma diagnostics by spacecraft electrostatic potential measurements, Ann. Geophysicae, 13, 118-129, 1995.

Persoon, A. M.: Electron density distributions in the high-latitude magnetosphere, Adv. Space Res., 8, 8, 79-88, 1988.

Persoon, A. M., Gurnett, D. A., Peterson, W. K., Waite, Jr., J. H., Burch, J. L., and Green, J. L.: Electron density depletions in the nightside auroral zone, J. Geophys. Res., 93, 1871-1895, 1988.

Scudder, J. D., Cao, X., and Mozer, F. S.: Photoemission current - spacecraft voltage relation: key to routine, quantitative lowenergy plasma measurements, J. Geophys. Res., 105, $21281-$ $21294,2000$.

Strangeway, R. J., Kepko, L., Elphic, R. C., et al.: FAST observations of VLF waves in the auroral zone: evidence of very low plasma densities, Geophys. Res. Lett., 25, 2013-2016, 1998.

Strangeway, R. J., Ergun, R. E., Carlson, C. W., McFadden, J. P., Delory, G. T., and Pritchett, E. L.: Accelerated electrons as the source of auroral kilometric radiation, Phys. Chem. Earth, 26, 1-3, 145-149, 2001. 